\title{
Using a scientific literacy cluster to determine participant attitudes in scientific events in Japan, and potential applications to improving science communication
}

\author{
Shishin Kawamoto, Minoru Nakayama, Miki Saijo
}

\begin{abstract}
Various science events including Science Cafés have been held in Japan. However, there is the question whether these are events in which all people in society can participate? In particular, methods for checking whether or not the event attracts the participants targeted by the organizers have not yet been well established. In this paper, the authors have designed a simplified questionnaire to identify the participants' attitudes toward science, technology and society, which can then be grouped into four clusters. When applied to various science cafés, the results revealed that participants consisted of Cluster 1 "Inquisitive type" and Cluster 2 "Sciencephile" who are interested in science and technology. The cafes studied did not provide sufficient appeal to people of Clusters 3 and 4 who are not interested in science and technology without applying some inventive methods. Our method provides a means of objectively evaluating the tendencies of participants in science communication events in order to improve the spread of science communications within society.
\end{abstract}

\section{Introduction}

The science café, which originated in the U.K., is different from conventional lectures or discussion meetings; it is a place where scientists and ordinary citizens communicate face to face. Its purpose has been defined as "We are committed to promoting public engagement with science and to making science accountable". Various kinds of science café are held in Japan, ${ }^{2}$ and in 2010, approximately 1,000 science cafés were held. ${ }^{3}$ Following their introduction in Japan by the government's "White paper on science and technology in 2004", 4 science cafés have spread and since 2005, have been held all over the country. In particular, during the Science and Technology Week established in April 2006 by the Ministry of Education, Culture, Sports, Science and Technology, science cafés were held at 21 places hosted by various universities, the Science Council of Japan, and by the Japan Science and Technology Agency under the co-sponsorship of the Ministry - thus accelerating the trend. As can be seen from this trend, the science café approach has been promoted by the 
government as a part of its science and technology policy; in addition, most of them are conducted by universities, research institutions and scientists. ${ }^{5}$

Because of this characteristic of Japanese science cafés, one wonders if they are really serving as places to encourage face to face communications between scientists and citizens. There is also the criticism that they tend to be held in a lecture style where in practice, science knowledge tends to be communicated uni-directionally. ${ }^{6}$ Even in the U.K., it has been pointed out that discussions in science communications tend to be on scientific facts rather than the ethical or political issues arising from science, and that the activities are more "provider driven" than "consumer driven". This is not to suggest that lectures are always inappropriate; there must be cases where lectures are appropriate for the intended purpose. However, we are questioning whether a science café is fully demonstrating its capability of reaching those people who have not been participating in conventional science events so far. In particular, we wonder if Japanese science cafés are becoming lectures for certain groups of citizens who are already interested in science and technology. While the object of the science café is the promotion of participation by citizens, this cannot be achieved if the participants are dominated by a certain group of people.

\section{Objective}

Not only science events including science cafés and lectures, but all communications are designed to have certain targets of participants. Although there are various definitions, Rogers as an innovation researcher defined a communication as "a process in which participants create and share information with one another to reach a mutual understanding". Since "the participants" have their own individual contexts, it is necessary to understand those and adapt the communication process accordingly if a smooth communication is to be achieved. Therefore, in order to design an event targeting unspecified numbers of people such as a science café, it is necessary not only to set an objective of what is intended to be gained by the participants, but also to clarify the following four points:

$1^{\text {st }}$ Point) What kind of attitudes toward science/technology and society do people have?

$2^{\text {nd }}$ Point) What kind of people should be targeted?

$3^{\text {rd }}$ Point) What kind of contents and format are needed to match the participants?

$4^{\text {th }}$ Point) What kind of people actually participated in science events?

Clarifying these points may enable us to define the target of communications, design communication channels, evaluate whether what is designed is achieved, and feedback results to the next communication. With such a cycle, a science café can be an event geared toward the original objective of promoting citizens' participation and contributing to societal accountability.

In order to clarify the first point (what percentage of people have what kind of attitudes toward science/technology and society), various large scale surveys are being carried out in the EU, ${ }^{9}$ U.S.A., ${ }^{10}$ and Japan. ${ }^{11}$ In addition, there are other kinds of surveys, such as the "Science and the Public" survey carried out by the U.K. Office of Science and Technology and the Wellcome Trust. ${ }^{7}$ These surveys classify people into a 
plurality of clusters based on their response patterns related to their attitudes toward science/technology, etc. They show the attributes and tendencies of attitudes on various themes related to science and technology for each cluster. We conducted a survey of people's attitude toward science/technology and society in Japan based on these surveys as well. ${ }^{12}$

These cluster surveys provide important insights concerning the process of targeting event participants, which is the second communication design point. They also provide some perceptions relevant to the process of making decisions on the content and configuration of events, which is the third point. Designing the place of communication, in particular how to present the issues and how to discuss them, is not an easy task even after the contents are decided. As to the design of the place of communication, the U.K.'s DANA Centre has proposed several styles of participation. $^{13}$

As can be seen from the above, the first through third points have been covered to a degree so far in previous studies. But few studies have, as far as we are aware, addressed the fourth point, i.e., checking what kinds of people have actually participated in particular events. In many science events, to evaluate the content and outcome of the event, a questionnaire survey is conducted after the event ends. In such questionnaires, in addition to evaluation items related to the contents of the event, respondents' genders and ages tend to be asked. ${ }^{14}$ However, such questionnaires do not clarify the general attitudes of the participants in science events toward science/technology and society. Consequently, we decided to develop a participant model to be used to specify target populations in various science events including science cafés. Additionally, we developed a method for identifying the tendencies of participants' attitudes toward science and technology at the time of the event.

\section{Methods}

\section{1. Scientific Literacy of Japanese}

In order to clarify the first point "What kind of attitudes toward science/technology and society do people have", we first conducted a large scale survey using questionnaires. Using a random selection method, we selected 4,000 respondents and sent out questionnaires by mail. A total of 1,286 responses were collected as a result. ${ }^{15}$ The results of responses to 1) interests in various fields including science and technology (15 questions), 2) attitudes and interests toward science and society (35 questions), and 3) evaluations on science/technology and society (15 questions) among the total of 98 questions included in the questionnaire were analyzed using factor analysis and cluster analysis to develop a model to assign Japanese people to a plurality of clusters exhibiting different tendencies in their attitudes toward science/technology and society. ${ }^{12}$ The extracted factors are the following three factors (for details of the questions representing factors, refer to table 1): 
$1^{\text {st }}$ factor) Scientific factor: Related to interests in science and technology, familiarity with using scientific/technological products, etc.

$2^{\text {nd }}$ factor) Social factor: Related to interests in local communities, welfare and environment, willingness to participate in social activities, etc.

$3^{\text {rd }}$ factor) Science-appreciating factor: Related to attitudes appreciating the values of science and technology and activities of scientists.

We clustered the data using these three factors, and developed a model consisting of 3 -factors and 4-clusters (figure 1). ${ }^{12}$ Cluster 1 was high in all factors. They have positive and optimistic attitudes toward science and society. Cluster 2 is a cluster with a high scientific factor, a low social factor, and a medium science-appreciating factor. The cluster has a large proportion of males, and a high percentage of young people. It would seem that constituents of Cluster 2 are more skeptical that those of Cluster 1. In Cluster 3 the scientific factor is low, the social factor is medium high, and the scienceappreciating factor is medium. A significant characteristic of this group is that it has a high proportion of women. Cluster 4 is low in all factors. Cluster 4 has a high percentage of women and moderately high percentage of young people.

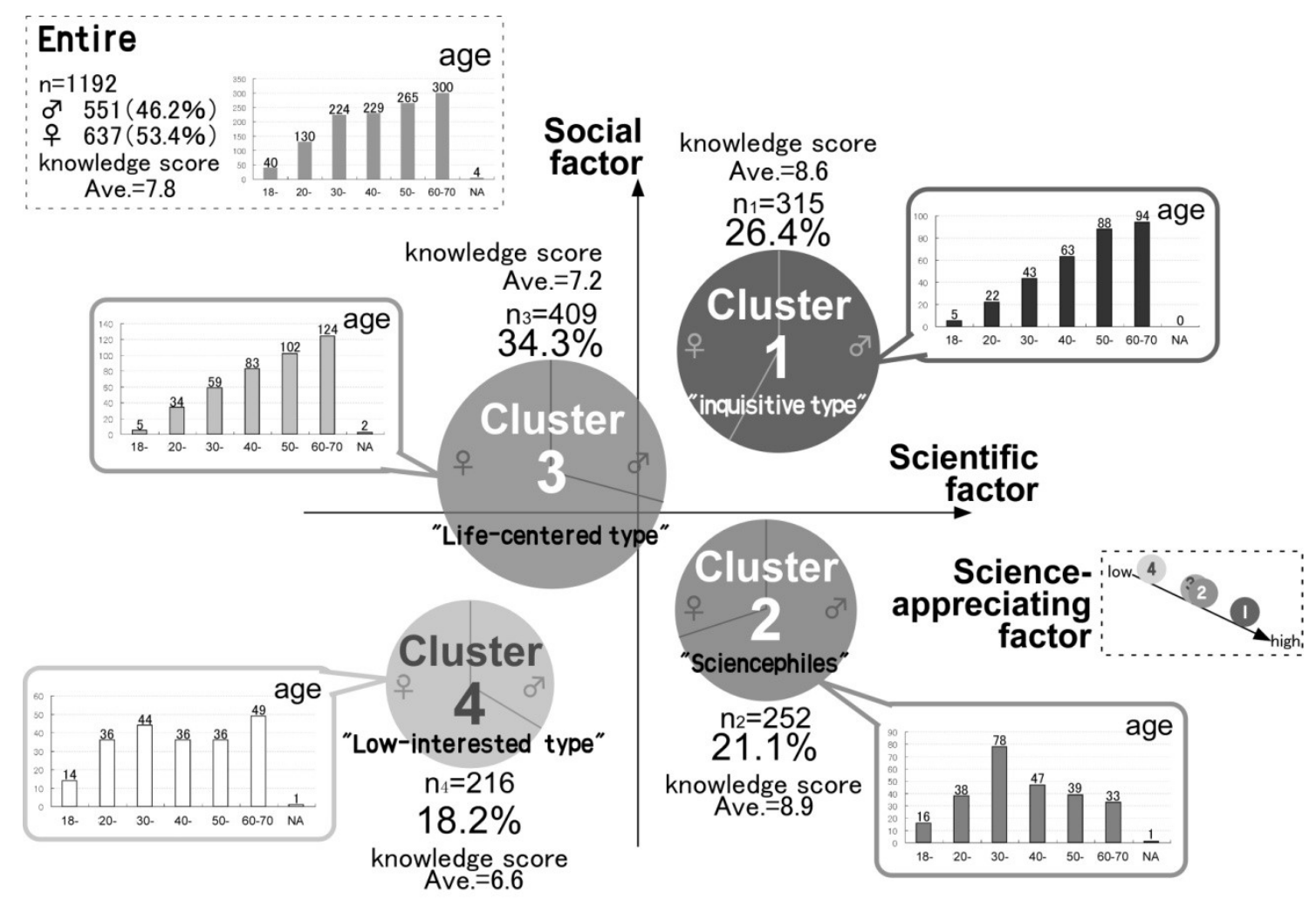

Figure 1. Outline of scientific literacy clusters in the random survey. Horizontal axis represents the scientific factor, vertical axis represents the social factor, and the colors of circles represent science-appreciating factors. Areas of circles represent populations. NA: No Answer. 


\begin{tabular}{|c||c|}
\hline $\begin{array}{c}\text { Factor } \\
\left(\mathrm{R}^{2}\right)\end{array}$ & \multicolumn{1}{c|}{ Contents } \\
\hline \hline $\begin{array}{c}\text { Scientific factor } \\
(0.86)\end{array}$ & Q1) I am knowledgeable of science and technology \\
& Q3) I wish to know more about science and technology \\
\hline $\begin{array}{c}\text { Social } \\
\text { factor } \\
(0.83)\end{array}$ & Q4) I am interested in the issue of local society \\
\hline $\begin{array}{c}\text { Science- } \\
\text { appreciating } \\
\text { factor }\end{array}$ & Q6) I am interested in the issue of culture \\
$(0.86)$ & Q9) I hope scientific thinking prevails more in the society \\
\hline
\end{tabular}

Answering: 4 step measuring (1: agree; 2: slightly agree; 3: slightly disagree; 4: disagree)

Table 1. Contents of simplified questionnaire.

\subsection{Preparation of Simplified Questionnaires}

In the next step, in order to clarify the fourth point "What kind of people actually participated in science events", we generated a simplified questionnaire to estimate a corresponding cluster for each respondent. This is due to the fact that the questionnaire we used in the national survey had many questions and was thus impractical for use with event participants at the time. In selecting the questions, we adopted a model (group of questions) consisting of a relatively small number of questions which showed high $\mathrm{R}^{2}$ values ${ }^{16}$ as a result of a multiple regression analysis using the stepwise method on the items used in the factor extraction together with each factor score.

The following regression formulae were obtained by multiple regression analysis. Independent variables $(\mathrm{x} 1 \sim 10)$ are the answer to each of the ten questions. Dependent variables $(y)$ are the factor score of each of the three factors.

Scientific factor:

$$
\begin{aligned}
& y_{\text {scif }}=3.438-0.433 x_{1}-0.433 x_{2}-0.415 x_{3} \\
& y_{\text {socf }}=2.923-0.426 x_{4}-0.451 x_{5}-0.325 x_{6}-0.29 x_{7} \\
& y_{\text {sciaf }}=2.817-0.741 x_{8}-0.391 x_{9}-0.36 x_{10}
\end{aligned}
$$

Social factor:

We calculated the distance between the three factor scores obtained by substituting the answers to the 10 questions and the four cluster centers obtained by the random mail survey (table 2). The respondent belongs to the nearest cluster. 


\begin{tabular}{|c||c|c|c|c|}
\hline & $\begin{array}{c}\text { Cluster 1 } \\
\text { Inquisitive }\end{array}$ & $\begin{array}{c}\text { Cluster 2 } \\
\text { Sciencephiles }\end{array}$ & $\begin{array}{c}\text { Cluster 3 } \\
\text { Life-centered }\end{array}$ & $\begin{array}{c}\text { Cluster 4 } \\
\text { Low interest }\end{array}$ \\
\hline \hline Scientific factor & 0.8216 & 0.7113 & -0.5195 & -1.0444 \\
\hline Social factor & 0.9403 & -0.5723 & 0.2205 & -1.1212 \\
\hline Science-app. factor & 0.9374 & -0.0256 & -0.1275 & -1.1554 \\
\hline
\end{tabular}

Table 2. Cluster centers.

We further conducted a discrimination analysis using the estimated value of the factor score in the multiple regression analysis as an independent variable and a cluster as a dependent variable, in order to obtain a matching rate for the cluster classification based on the small number of questions and the cluster classification based on the original data. As a result, we obtained matching rates of $92.4 \%$ for Cluster $1,90.9 \%$ for Cluster 2, 93.6\% for Cluster 3, and 94.9\% for Cluster 4. From this, we concluded that the simplified questionnaire is effective.

In addition to the 10 questions, we added questions on the respondent's age and gender in the actual questionnaire. We also provided an area for additional questions to be added as needed in a particular event or where respondents can write freely, e.g. to make individual comments or requests.

\section{Results}

The above simplified questionnaire was applied in science cafés and science events in which the general public participated, together with scientists and graduate students from our university (figure 2). We could evaluate the tendencies of the participants by comparing the cluster distribution at each event with that of the national random survey. The results of each survey are described below.

Votes that could not be classified due to incomplete responses are omitted. The graphs on the right show gender comparison. Case A was a survey using complete questionnaires, while others were surveys using the simplified version consisting of 10 questions. The event theme, targeted population, date and place of survey for A-P cases are as shown below. The values shown at the end represent response rates and evaluation results in comparison with cluster shares of case $\mathrm{A}\left(\chi^{2}\right.$ test P-value. $\square$ statistically insignificant; $* 0.001<\mathrm{P}<0.05 ; * * 0.001<\mathrm{P}<0.01 ; * * * \mathrm{P}<0.001)$. NA: No Answer.

A) Random selection postal survey (March 18 - April 7, 2008, all over Japan) $32.2 \%$

B) Participants in a science café on reintroduction of Toki (Crested ibis) and farming (" $16^{\text {th }}$ Science café Niigata" November 29, 2008, Niigata) $100 \%$, $P=0.001$

C) Participants in a science café on automata and beauty (" 7 th $T o k y o$ Tech Science café" January 39, 2010, Tokyo) $100 \%, P=0.001$

D) Participants in a science café on virus disease and farming $\left(5^{\text {th }}\right.$ HokuNouKen café" August 7, 2010, Sapporo) 100\%, $P=0.000$ 
E) Participants in a science café on super cooling in pub (" 6 th Tokyo Tech café" January 31,2009 , Tokyo) $100 \%, P=0.000$

F) Participants in a science café on art in dining bar (" 2 nd Science \& Culture café" March 28, 2009, Tokyo) $100 \%, P=0.000$

G) Participants of a science café on solar energy and culture (" $12^{\text {th }}$ Creative café" July 31, 2011, Tokyo) $100 \%, P=0.007$

H) Participants in a science café on radio building (" $18^{\text {th }}$ Science café Hiroshima" January 30, 2010, Hiroshima) Collection rate unknown, $P=0.198$

I) Participants in a science café on fragrance (" 8 th Tokyo Tech café" January 22, 2011, Tokyo) $100 \%, P=0.057$

J) Participants in a science experiment event for parents and children on fuel cells (December 19, 2009) Approximately 100\%, $P=0.035$

K) Visitors to the booth exhibiting scientific literacy at Science Agora 2008 (November 22-24, 2008, Tokyo) Approx. 100\%, $P=0.000$

L) Participants at a talk event on fuel cells presented at Science Agora 2009 (October 31, 2009, Tokyo) Approx. 60\%, $P=0.000$

M) Graduate students who took a science communication education class of Tokyo Institute of Technology (2008-2011, Tokyo) 94.1\%, $P=0.000$

N) Education professionals who attended a science education workshop (June 25, 2009, Tokyo) Approx. $100 \%, P=0.003$

o) Volunteer citizens and others who attended a science communication education workshop (February 3, 2009, Hiroshima) 100\%, $P=0.000$

P) Science researchers who attended a lecture related to scientific literacy of a energy science forum (December 15, 2008, Nikko) Approx. 100\%, $P=0.000$

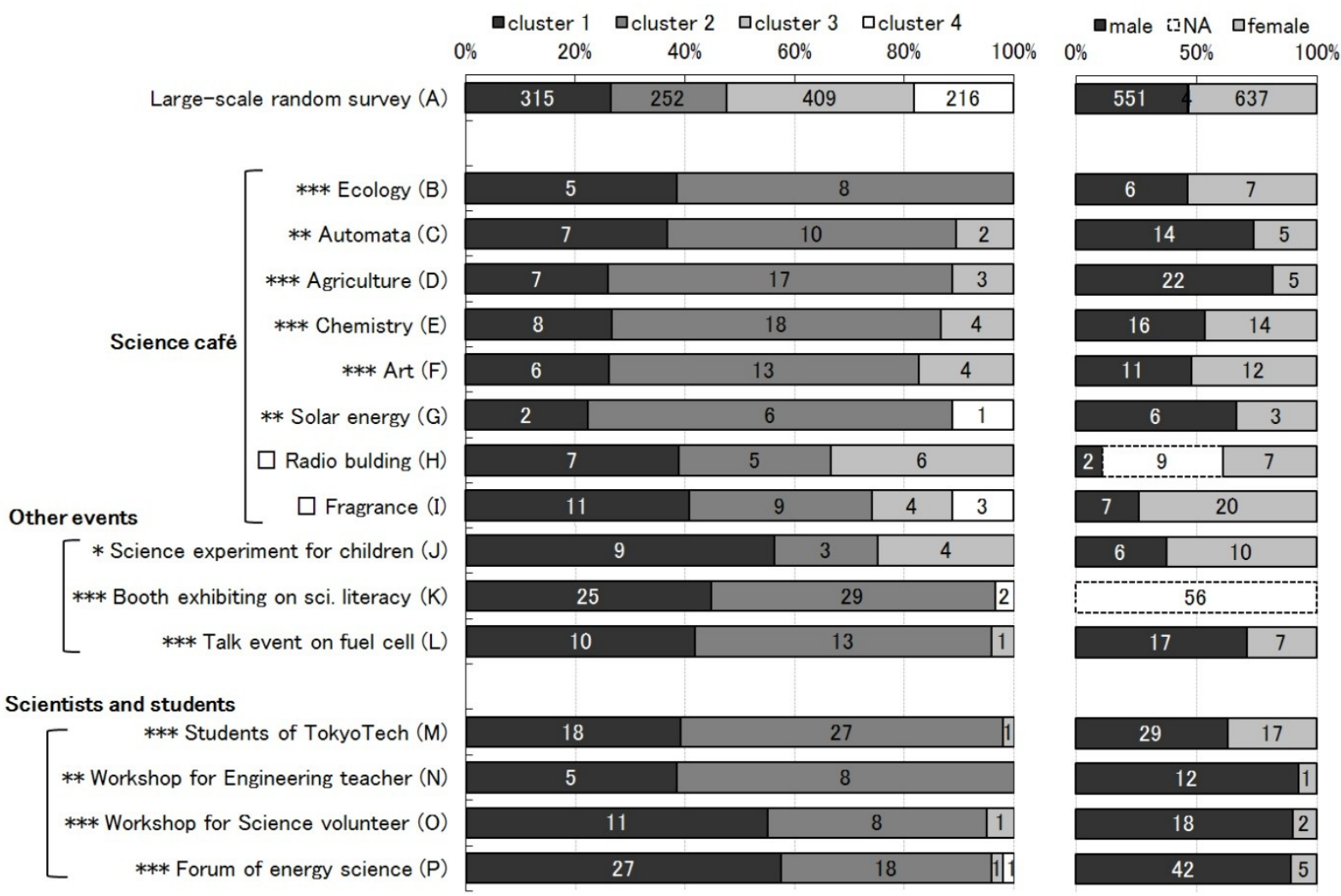

Figure 2. Comparison of cluster distributions between the national survey and the surveys in science events. 
Events (B) through (L) in figure 2 show cluster tendencies in the participants in science cafés and other events designed for non-professional participants. Events (B) through (I) in figure 2 are the results of surveys conducted in various science cafés. In science café (B), the participants consisted primarily of Clusters 1 and 2. However, in cafés (C)-(I), approximately $30-90 \%$ of the participants belonged to either Cluster 1 or Cluster 2, but some of them belonged to Cluster 3 and Cluster 4. Especially, in café $(\mathrm{H}), 33 \%$ of participants belonged to Cluster 3. In café (I), $14.8 \%$ belonged to Cluster 3 , and $11.1 \%$ to Cluster 4 . In the survey in event $(\mathrm{J})$, which was carried out with parents who participated in a science experiment event intended for children and their parents, $18.8 \%$ of them belonged to Cluster 3 and $25 \%$ to Cluster 4.

Events (K) and (L) were held in Science Agora which is the largest festival of science and technology in Japan. ${ }^{17}$ Although this is different from the British Science Festival held in the U.K. and sponsored by the British Science Association or the AAAS Annual Meeting in U.S., it is the largest science communication event for professionals and nonprofessionals in Japan and sponsored by a government agency, and thus is representative of the present condition of science communication in Japan. Although the data shown in $(\mathrm{K})$ and $(\mathrm{L})$ are not surveys conducted for all the participants of Science Agora, but rather the results of surveys conducted on participants in our own events, they show that the participants consisted almost exclusively of Clusters 1 and 2.

The cases shown in $(\mathrm{M})$ through $(\mathrm{P})$ represent the cluster tendency of students and professionals in science and technology. The participants primarily belonged to Clusters 1 and 2. (M) "Science and Engineering Communication" is a subject of the graduate school at Tokyo Institute of Technology where students were mostly in their 20 s, so that Cluster 2 (primarily of young ages) shared $58.7 \%$ of the entire population. $(\mathrm{N})$ and $(\mathrm{O})$ in figure 2 were intended for people who are engaged in education and activities related to science communications. The cluster compositions of these events were also characterized by the majority of the participants belonging to Clusters 1 and 2. (P) was intended for researchers related to energy science and engineering. The cluster composition of this group was similar to the ratio in event $(\mathrm{O})$ in that Clusters 1 and 2 comprised the majority of the participants, but a difference was observed in that Cluster 1 membership was larger and Cluster 2 membership was smaller relative to event (M) where the majority of the participants were students. From these results, it was suggested that people organizing science communication events and providing pertinent information tend to belong to Clusters 1 and 2, while younger people tend to belong primarily to Cluster 2 .

From these results, it is suggested that scientists and natural science students can be classified as either Cluster 1 "Inquisitive type" or "Sciencephiles" (M-P in figure 2), and the participants in the events they organize also tend to be Cluster 1 or 2 (B-L). On the other hand, some of the participants in science cafés and the science experiment event intended for children and their parents provided in various science events aimed at the general public, were found to belong to Cluster 3 "Life-centered type" (C-F, HJ). However, the presence of Cluster 4 was extremely rare in the science events that we surveyed. 


\section{Discussion}

These survey results thus suggest that even an event "intended for general public" results in most of the participants consisting of those belonging to Clusters 1 and 2. Of course, it is not a problem for a science event to have participants mostly consisting of Clusters 1 and 2. In particular, it presents no problem if the event is designed from the start as an event intended for people of Clusters 1 and 2. However, each event organizer and promoter should think carefully about the second point "What kind of people should be targeted?". As discussed in the beginning of this paper, we need events where people from Clusters 3 and 4 can participate, if the purpose of science café is to promote participation of citizens in science and to let science fulfill its accountability to society. It is difficult to conclude that heterophily ${ }^{8}$ - the degree to which people who interact are different in certain attributes - is sufficiently secured in today's science communications in Japan. In Japan, science cafés were promoted as a tool of science and technology policies, and most of them are conducted by people in scientific fields. Generally, targeting people who have different attitudes from oneself makes it more difficult to design attractive events, and to advertise such events through effective communication channels. This seems to be one reason why almost all participants at science café belong to Cluster 1 and Cluster 2; the same as people in scientific fields.

Our results raise the question what kind of science event can attract both Clusters 1 and 2 and Clusters 3 and 4, and thus attract participants who cross the gaps in communication and secure high heterophily? This is the answer to the third point "What kind of contents and format are needed to match the participants?" We cannot draw a detailed conclusion because the number of cases available is too small, and the methodology for analyzing event contents has yet to be established. However, some common characteristics were noted in the events where there were significant numbers of participants outside the range of Clusters 1 and 2 .

First, one favorable factor appears to be where events' main themes and participants are connected only indirectly to science and technology. One example of such a case was the science café where the main theme was art into which scientific and technological topics were embedded (figure 2C, F); a second example was the science experiment event for parents and children where, although the main target was children, their parents nevertheless experienced the same scientific experiments indirectly (figure $2 \mathrm{~J}$ ).

Secondly, there appears to be a favorable impact where events use publicity methods which have high appeal to the intended participants. In one case, a bar was used as the venue for a science café, by putting up posters in the bar's neighborhood and the nearby railway station; this succeeded in attracting many bar customers (figure 2E, F). Another example was the Science Café Hiroshima (figure 2H). Hiroshima Municipal Science and Technology Citizen's Council, which is a volunteer group that organized this café, used a public relations paper ("Citizens of Hiroshima and Municipal Administration") to advertise the café. This PR paper is issued to all residents twice a month, and thus provides a powerful vehicle for public announcements; this appears to have helped the science café to appeal to a wide range of participants belonging to all 
four clusters. Using such information dissemination through the municipal government can give promoters confidence that their activity will be effectively carried out.

Thirdly, one science café was targeted at women (figure 2I), with the theme of fragrance. To attract women's' interest, female students designed the media relations which included handbills and website announcements. This approach proved very successful: $74.1 \%$ of participants were women, and many participants belonged to Cluster 3 and 4. All the participants in Clusters 3 and Cluster 4 were women; one woman in Cluster 3 and two women in Cluster 4 were teenagers. Therefore, to target participants in Clusters 3 and 4, one approach would be to design an event for young women.

Nevertheless, none of the events surveyed attracted Cluster 4 participants as the majority. Moreover, the scientific literacy model consisting of three factors and four clusters we originally designed detected Cluster 4 as only a residual cluster, and was thus not able to determine the interests or concerns of people belonging to Cluster 4 . It is important to further understand the interests of people belonging to Cluster 4 and find ways of prompting them to participate in pertinent communications, if we are to aim to raise the entire population's scientific literacy.

\section{Conclusions}

What we are aiming for in scientific literacy improvement is the improvement of scientific literacy of the whole of society. Our cluster model is not intended to converge the population into a specific cluster. For example, we do not advocate seeking to encourage all people to change to cluster 1 characteristics. Rather, this model is intended as a tool for us in science communication activities in order to investigate whether the participants are biased to a particular cluster. We consider that it is important to encourage a mixture of participants from different clusters in science communication events, such as science cafes. From this perspective, our cluster model can provide a useful approach in the targeting of participants.

We are currently conducting educational programs aimed at improving scientific literacy through promoting communication between the clusters. ${ }^{18}$ For example, we have a program for executing a science café organized by natural science students (who primarily belong to Cluster 2) aimed at participants of Cluster 3 women; and to promote discussions on cultural and trans-scientific topics. In such a space, while recognizing the differences between various interests and ways of thinking, we can think of the aim of science communication as being to bring various trends in literacy to form a 'collective scientific literacy'.

However, it is difficult to design communication venues of high heterophily. Without accumulation of these research methods and knowledge, we will not be able to approach our goal of realizing citizens' participation, rather than having science communication ending up as a transient activity. It is necessary from now on to conduct surveys on further varieties of events, accumulate classification and analysis data related to event contents, and advance studies on what kind of themes and topics are attractive to each Cluster (particularly Cluster 3 and Cluster 4), and how to design optimal venues and methods of communication. 


\section{Acknowledgment}

Our heartfelt gratitude goes to Science café Niigata (Representative: Associate Prof. Yoshio HONMA), HokuNouKen café (Representative: fellow researcher Takuro SHINANO), Creative café (Representative: Associate Prof. Kayoko NOHARA) Science café Hiroshima (Representative: Prof. Takehiro HAYASHI) and all the people who assisted us in this survey. We appreciate generous support from prof. Michael Norton.

\section{Notes and references}

${ }^{1}$ Café Scientifique, http://www.caféscientifique.org/.

2 M. Nakamura (2008), Science Café: its scope and challenge (in Japanese), Journal of Science and Technology Studies 5: 31-43.

${ }^{3}$ Ministry of Education, Culture, Sports, Science and Technology (2011), White paper on science and technology 2011: create science and technology with society (in Japanese), National Printing Bureau, pg. 82.

${ }^{4}$ Ministry of Education, Culture, Sports, Science and Technology (2004), White paper on science and technology 2004: science and technology and society in the future (in Japanese), National Printing Bureau, pg. 88-133.

${ }^{5}$ For example, Matsuda (2008) reported 72 science cafés which were held in Japan in 2007. ${ }^{6}$ Of those, 39 were held by universities and research institutions, and of those 10 were held by groups of students and scientists.

${ }^{6} \mathrm{~K}$. Matsuda (2008), Science café in japan: a report of the poster exhibition and the workshop about science café in science agora 2007 (in Japanese), Japanese Journal of Science Communication 3: 3-15.

${ }^{7}$ Office of Science and Technology and the Wellcome Trust (2000), Science and the public: a review of science communication and public attitude to science in Britain.

${ }^{8}$ E.M. Rogers (2003), Diffusion of innovations, fifth edition, Free Press.

${ }^{9}$ European Commission (2010), Special Report: Science and Technology, Special Eurobarometer $340 /$ Wave 73.1, http:/lec.europa.eu/public opinion/archives/ebs/ebs 340 en.pdf.

${ }^{10}$ National Science Foundation (2010), Science and engineering indicators 2010.

${ }^{11}$ National Institute of Science and Technology Policy, Ministry of Education, Culture, Sports, Science and Technology (2002), Summary: The 2001 survey of public attitudes toward and understanding of science \& technology in japan, NISTEP report no. 72.

${ }^{12}$ S. Kawamoto, M. Nakayama and M. Saijo (2012, in press), A survey of scientific literacy to provide a foundation for designing science communication in Japan, Public Understanding of Science.

${ }^{13}$ Dana centre - event styles, http://www.danacentre.org.uk/aboutus/eventstyles.

${ }^{14}$ For example, Japanese Journal of Science Communication 1-5, 7-9 (2007-2011) reports fourteen science events of science café or similar format; in all of those cases, either a selection type or free description type of questionnaire were conducted.

${ }^{15} \mathrm{We}$ conducted a two-stage stratified random sampling from the Basic Resident Resisters. In the first stage, the whole of Japan was divided into 10 regions, and in the second stage, these regions were further divided into 3 urban groupings. The total 30 groupings were then divided into 200 areas. Finally we made a random selection of 20 people from each of the 200 areas. Refer to note 12 for the details.

${ }^{16} \mathrm{R}^{2}$ value (coefficient of determination) provides a measure of how well outcomes are likely to be predicted by the model. The value is a measure between zero and one. The higher value signifies higher predictive capability.

${ }^{17}$ Science Agora, http://www.scienceagora.org/scienceagora/agora2009/index_e.html.

${ }^{18}$ M. Norton, K. Nohara (2009), Science cafés. Cross-cultural adaptation and educational applications, $\operatorname{JCOM~8(4):~1-12.~}$ 


\section{Authors}

Kawamoto Shishin is a research associate at the Tokyo Institute of Technology. After completing his Ph.D. in developmental biology, he focused on surveys of scientific literacy and education in science communication. Address: Graduate School of Engineering, Department of Mechanical and Control Engineering, Tokyo Institute of Technology, 2-12-1 NE-20 Ookayama, Meguro-ku, Tokyo, JAPAN, 152-8550. E-mail: kawamoto.s.aa@m.titech.ac.jp.

Nakayama Minoru is a professor at the Tokyo Institute of Technology. He is analyzing the relation between the characteristics found in human learning behavior and the environment a learner is placed in using engineering methods. Address: Center for Research and Development of Educational Technology, Tokyo Institute of Technology, 2-12-1 W9-107 Ookayama, Meguro-ku, Tokyo, JAPAN, 152-8550. E-mail: nakayama@cradle.titech.ac.jp.

Saijo Miki is a professor at the Tokyo Institute of Technology. Her professional field of study is Applied Linguistics and the central theme of her study is formal communication between people with different knowledge and experiences. Address: International student center, Tokyo Institute of Technology, 2-12-1 NE-19 Ookayama, Meguro-ku, Tokyo, JAPAN, 152-8550. E-mail: msaijo@,ryu.titech.ac.jp.

How TO CITE: S. Kawamoto, M. Nakayama and M. Saijo, Using a scientific literacy cluster to determine participant attitudes in scientific events in Japan, and potential applications to improving science communication, JCOM 12(01) (2013) A01. 